\title{
A Rare Case of Neck Pain: Acute Longus Colli Calcific Tendinitis in a Possibly Immunocompromised Individual
}

\author{
Kerlie Estimable, MD, Cynthia Rizk, MD, and George G. A. Pujalte, MD
}

We present a rare case of severe neck pain in a 45-year-old man with severe hidradenitis suppurativa who was participating in a study involving adalimumab. The neck pain was associated with acute longus colli calcific tendinitis, which is a noninfectious inflammatory response in the longus colli tendons secondary to deposition of calcium hydroxyapatite crystal. The diagnosis was made by computed tomography, which showed calcifications and deposits, and magnetic resonance imaging, which showed a retropharyngeal effusion. Ears, Nose, and Throat Services performed a fiberoptic scope examination, which revealed a patent airway and no drainable abscess. Nonsteroidal anti-inflammatory drugs resulted in a dramatic improvement in the patient's clinical symptoms. In acute longus colli tendinitis, differentiating retropharyngeal aseptic effusion from infection is important. of note, the confounding factor in this case was that the patient was blinded to whether he was receiving the placebo or adalimumab, so whether the patient was immunosuppressed and at risk for infection was unknown. Clinician familiarity and education concerning acute calcific longus colli tendinitis may lead to decreased costs stemming from incorrect diagnosis and unnecessary treatment. (J Am Board Fam Med 2015;28:146-150.)

Keywords: Neck Pain, Tendinitis

Acute calcific tendinitis of the longus colli muscle is a rare condition that causes severe neck pain. The clinical presentation is commonly mistaken for other life-threatening conditions, such as neck abscess, trauma, cervical disk herniation, and neoplasm. ${ }^{1}$ Symptoms are nonspecific and include odynophagia, stiffness, neck pain, mild fever, and limitation of cervical motion. Pertinent laboratory values may include elevation of erythrocyte sedimentation rate, C-reactive protein, and white blood cell count. ${ }^{1,2}$

Acute calcific tendinitis of the longus colli is presumed to arise from a noninfectious inflamma-

This article was externally peer reviewed.

Submitted 21 April 2014; revised 9 August 2014; accepted 13 August 2014.

From the Departments of Family and Community Medicine (KE, CR, GGAP) and Orthopaedics and Rehabilitation (GGAP), Penn State Milton S. Hershey Medical Center, Hershey, PA.

Funding: none.

Conflict of interest: none declared.

Corresponding author: Kerlie Estimable, MD, Penn State Hershey Family Medicine Residency, 4 Williamsburg Bldg, Hershey, PA 17033 (E-mail: kestimable@yahoo.com). tory response of the superior oblique tendons of the longus colli muscles secondary to deposition of amorphous calcium hydroxyapatite crystal at the anterior C1-C2 disk space; however, some cases occur in more unusual areas (eg, C5-C6). ${ }^{2,3}$ Additional features seen on imaging include a nonsuppurative retropharyngeal effusion. Plain radiography is the first line in diagnosis, but computed tomography (CT) and/or magnetic resonance imaging (MRI) are the primary imaging methods used for definitive diagnosis. ${ }^{3,4}$

We present a unique case of acute calcific longus colli tendinitis in a patient, concurrently enrolled in an adalimumab trial, who had incidental findings of rotatory subluxation of $\mathrm{C} 3-\mathrm{C} 4$. Both considerations delayed confirmation of the correct diagnosis.

\section{Case Report}

\section{History}

A 45-year-old male football coach presented with a 3-day history of abrupt onset of sharp posterior cervical neck pain. He described it as severe pain 
Figure 1. Lateral view of the cervical plain radiograph showed a large area of prevertebral soft-tissue swelling (long arrows) from C1 to C5. Focal calcification was noted inferior to the $\mathrm{C} 1$ arch (short arrow).

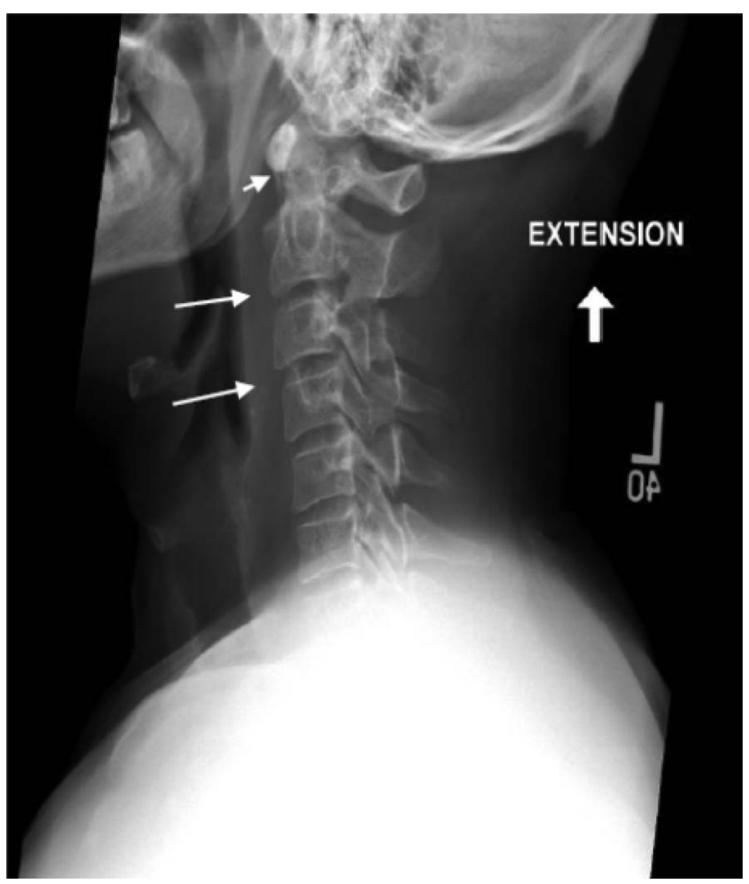

"deep" into his neck, radiating up to his temples, and exacerbated by turning his head to the left. He also had decreased neck range of motion, difficulty opening his jaw, and swallowing due to pain. With each attempt to swallow, he felt a pulling sensation and increased pain. He took ibuprofen and tramadol, which helped minimally. He reported 2 to 3 episodes of neck stiffness every year, but these were usually self-limiting. His medical history was significant for severe hidradenitis suppurativa, for which he was participating in a study investigating adalimumab as a treatment option compared with placebo. He was blinded to the arm to which he was assigned.

\section{Physical Examination}

The patient's vitals were as follows: blood pressure, 140/100 $\mathrm{mmHg}$; pulse rate, 100 beats/min; respiratory rate, 12 breaths/min; oxygen saturation, $98 \%$ on room air; and temperature, $36.8^{\circ} \mathrm{C}$. The patient was sitting uncomfortably on the table with a very stiff neck posture. Neck range of motion was decreased; he had $<10$ degrees of flexion, extension, and ear-to-shoulder movement bilaterally and was able to open his mouth only about $50 \%$. He had slight halitosis, but no oral lesions or palpable adenopathy were noted. Cardiorespiratory, abdominal, musculoskeletal, and neurological examinations were all within normal limits. His white blood cell count was mildly elevated at $12.85 \mathrm{~K} / \mu \mathrm{L}$. Both the erythrocyte sedimentation rate $(49 \mathrm{~mm} /$ hour $)$ and C-reactive protein $(7.06 \mathrm{mg} / \mathrm{dL})$ also were elevated.

\section{Emergency Department Course}

Cervical spine radiographs showed prevertebral soft-tissue swelling from C1 to C5 (Figure 1). CT of the cervical spine revealed calcifications anterior to the $\mathrm{C} 1-\mathrm{C} 2$ level and a retropharyngeal softtissue collection extending from $\mathrm{C} 2$ to $\mathrm{C} 4$ (Figures 2 and 3). Orthopedic Surgery was consulted because a defect also was seen at $\mathrm{C} 3$. The orthopedist felt that this was a congenital slight rotatory subluxation of $\mathrm{C} 3-\mathrm{C} 4$ (not visualized in the figures). Ears, Nose, and Throat (ENT) Services were consulted and, using a fiberoptic scope, noted a patent airway and no drainable abscess, determining that the patient had a retropharyngeal phlegmon and should be admitted for intravenous (IV) antibiotics. He was admitted, and a neck MRI was ordered.

Figure 2. Sagittal computed tomography scan shows an amorphous calcification (short arrow) anterior to the C1-C2 level. The disk space and heights were maintained. A retropharyngeal soft-tissue fluid collection extends from C2-C4 (long arrows).

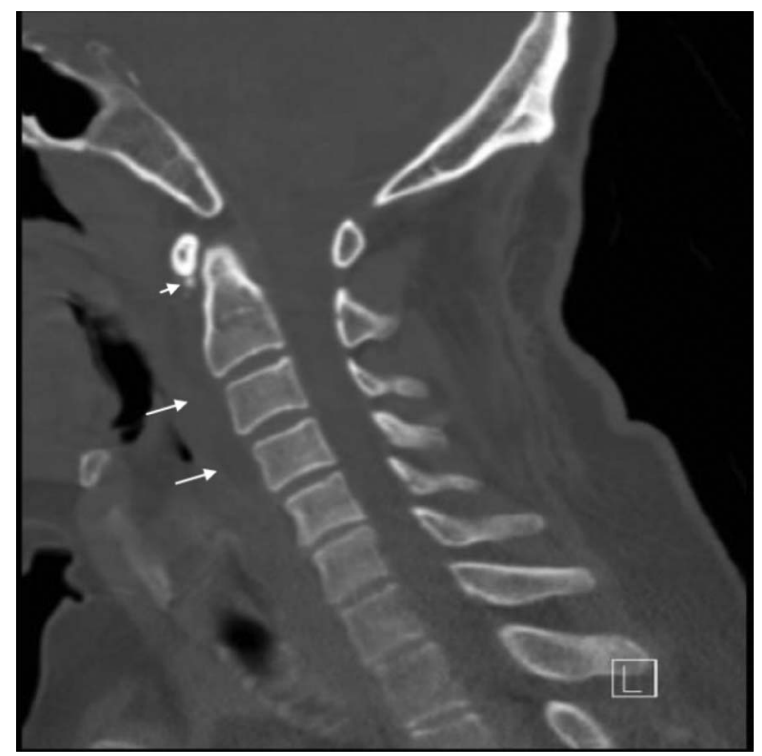


Figure 3. Computed tomography of the axial neck shows a calcific density (arrow) at the superior tendons of the longus colli at the C1-C2 level.

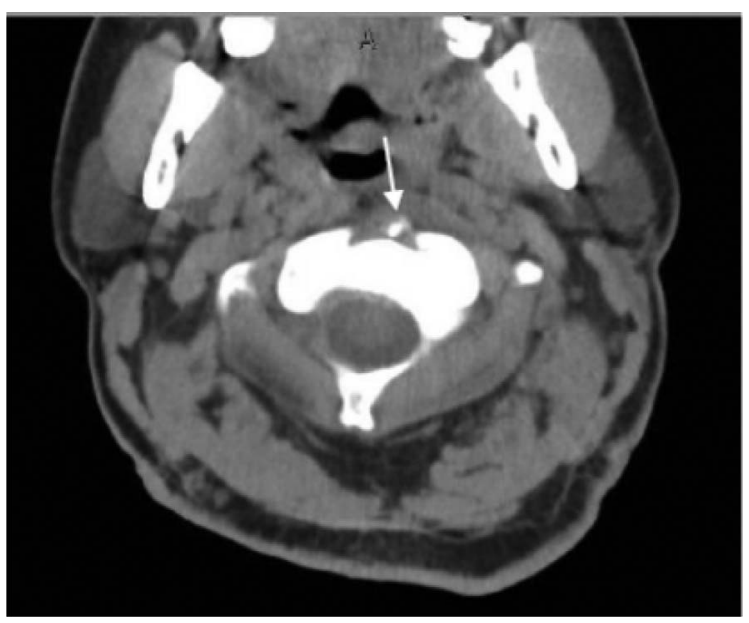

\section{Hospital Course}

The differential diagnoses were cervical torticollis, possible trauma, abscess, spontaneous retropharyngeal phlegmon, and deep neck infection related to adverse effects of adalimumab. The working diagnosis was retropharyngeal phlegmon since no drainable abscess was found, but the patient was given IV ampicillin/sulbactam to address a possible deep neck tissue infection given his involvement with the adalimumab trial. Hydromorphone and cyclobenzaprine were given for pain control, with no significant improvement. MRI showed a focal, nonenhancing wall effusion dissecting bilaterally into the longus colli muscles within the retropharyngeal space, extending from C1 to C5 (Figures 4 and 5). The diagnosis favored acute calcific longus colli tendinitis, particularly because focal calcification of the longus colli muscle at the C1-C2 level was previously noted on the CT scan.

The Orthopedic Surgery service also reviewed the MRI findings and recommended IV steroids and inpatient physical therapy. The patient's adalimumab study supervisor was contacted; the primary investigator was on vacation, so whether the patient was in the adalimumab or the placebo arm of the study could not be determined.

A literature search for acute calcific longus colli tendinitis was conducted, and few cases were found. Some recommended nonsteroidal anti-inflammatory drugs. Ketorolac $(15 \mathrm{mg})$ given every 6 hours drastically improved the patient's condition by the next day. He was discharged after 3 days with a
Figure 4. Sagittal view of a $\mathrm{T}_{1}$-weighted magnetic resonance image shows a bright retropharyngeal space focal effusion (arrow) dissecting into the longus colli muscles bilaterally from the anterior C1-C5 level, the absence of wall enhancement in the surrounding soft tissue, and the absence of suppurative retropharyngeal lymph nodes.

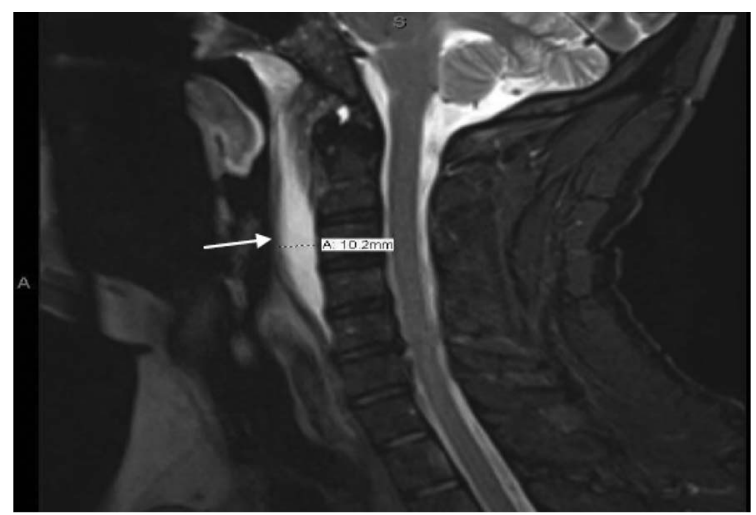

prescription to take diclofenac sodium $50 \mathrm{mg}$ by mouth 3 times a day as needed for 10 days. Ampicillin/sulbactam was switched to amoxicillin/clavulanate to be taken for 10 days in total.

\section{Follow-up After Treatment}

One week after treatment the patient was doing well, able to tolerate a regular diet, and move his neck without pain. Seven weeks later he continued to be asymptomatic. He was still enrolled in the

Figure 5. Additional sagittal view shows a bright retropharyngeal space focal effusion (arrow) dissecting into the longus colli muscles bilaterally from the anterior $\mathrm{C1}-\mathrm{C} 5$ level.

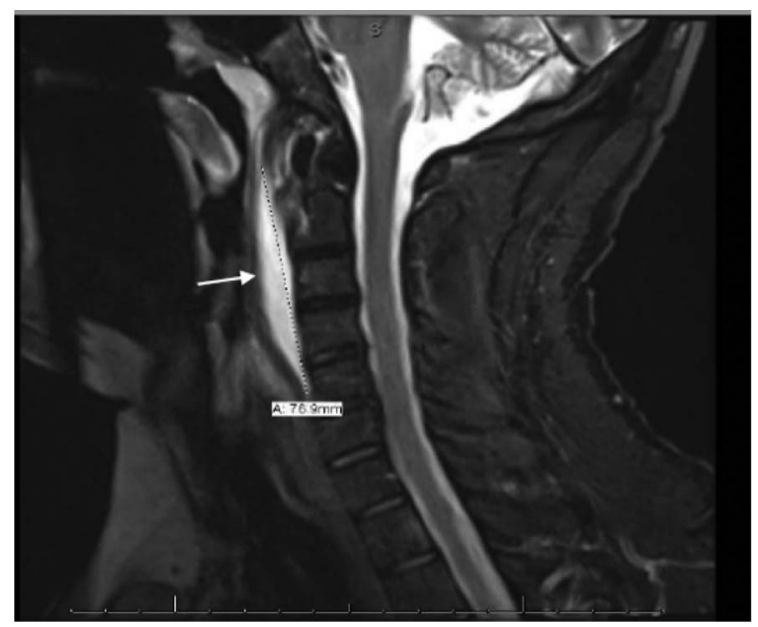


Figure 6. $T_{2}$-weighted magnetic resonance image shows a bright retropharyngeal effusion (arrow).

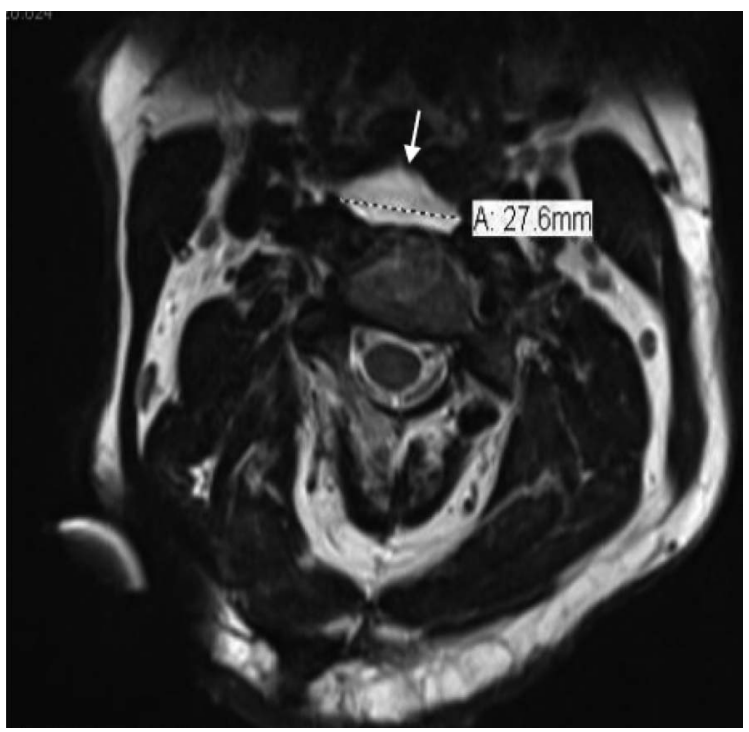

adalimumab trial and he had completed the abovementioned treatment course. Repeat MRI showed significant improvement of the prevertebral, nonenhancing walled fluid collection (Figure 7). Based on a phone follow-up a couple of months later, the patient had completed the adalimumab trial without recurrence of neck pain. It was confirmed that patient has been received adalimumab and not the placebo.

\section{Discussion}

The uniqueness of this case stems from the fact that our patient was at a potentially higher risk for infection because of his involvement in a trial of adalimumab for hidradenitis suppurativa. Adalimumab is a potent antagonist of tumor necrosis factor biological activity. ${ }^{5}$ The US Food and Drug Administration warns that adalimumab may put patients at higher risk of serious infections. ${ }^{5} \mathrm{Be}-$ cause we did not know to which arm of treatment the patient was assigned, the decision was made to initiate antibiotics to address any possible infectious component to his condition.

In our patient acute calcific tendinitis of the longus colli muscle was accompanied by a large retropharyngeal space effusion and amorphous calcium deposit at the $\mathrm{C} 1-\mathrm{C} 2$ level of the cervical spine. MRI revealed a nonenhancing wall effusion within the retropharyngeal space extending from C1-C5, without suppurative inflammation (Figures
4-6). The final diagnosis then was confirmed as acute calcific tendinitis rather than an infectious process. This condition is also known as acute calcific prevertebral tendinitis or calcific retropharyngeal tendinitis.

The incidence of acute calcific longus colli tendinitis is still unknown because the diagnosis is frequently missed. ${ }^{1,2,6}$ The disease occurs in both sexes, with a slight female predominance. It normally occurs in the third to sixth decades of life. ${ }^{4,6}$ Since it was first recognized, research about this entity has depended primarily on case reports. ${ }^{6}$ The first case reports were described in the 1960s. ${ }^{6,7}$ Later, in the 1990s, the calcifications were identified as amorphous hydroxyapatite deposits within the superior tendons of the longus colli. ${ }^{8,9}$

Initial testing includes lateral neck radiography; however, visualizing the calcification is difficult. ${ }^{10}$ CT of the cervical spine is the standard because it is more sensitive for demonstration of calcium hydroxyapatite deposits. ${ }^{10}$ Compared with CT, MRI is superior in delineating edema along the retropharyngeal space but is inferior in terms of demonstrating calcification. ${ }^{2,3,10}$ Retropharyngeal space effusions have been reported in some case reports similar to ours, ${ }^{2,4}$ but there has been no report in a

Figure 7. Sagittal view of a $\mathbf{T}_{1}$-weighted magnetic resonance image shows interval resolution (arrow) of the prevertebral enhancing fluid collection, with no evidence of abscess or residual inflammation.

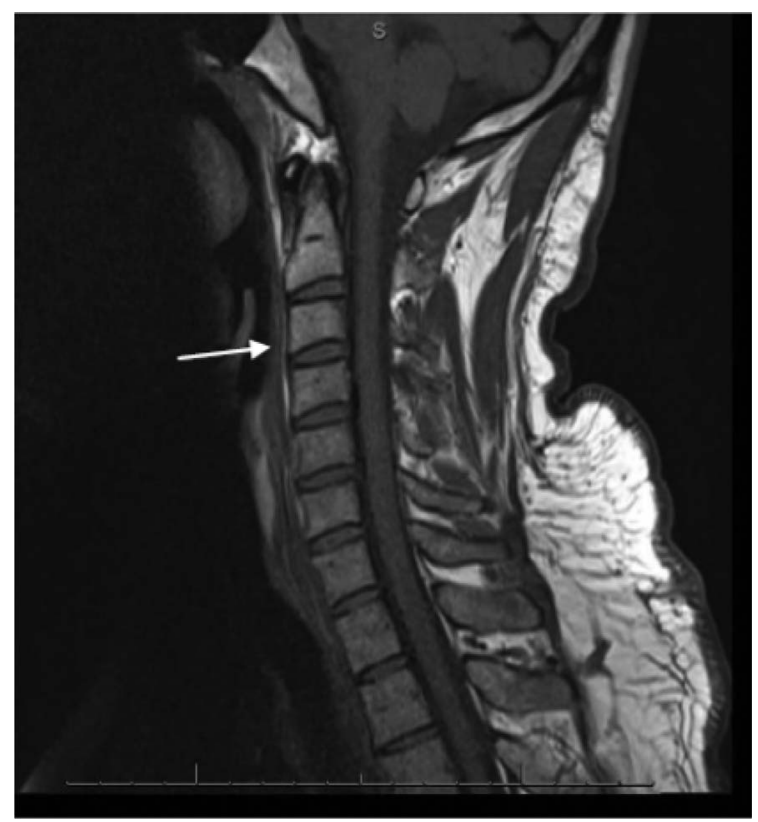


patient at high risk for disseminated infection, thereby making an abscess a concurrent possibility.

There are 2 ways to differentiate a retropharyngeal space effusion from an abscess. First, there must be no surrounding enhancement of the effusion. Second, suppurative inflammatory retropharyngeal lymph nodes must be absent. ${ }^{3,4,8,10}$ Eastwood et $\mathrm{al}^{11}$ reported a similar description to differentiate a retropharyngeal space effusion from an abscess. Our patient's MRI pointed toward an effusion rather than an abscess.

Acute calcific tendinitis of the longus colli muscle is a self-limiting condition. ${ }^{2,3,6,9-12}$ The first line of treatment is nonsteroidal anti-inflammatory drugs. ${ }^{1-12}$ In severe, painful episodes patients may also benefit from corticosteroids. ${ }^{2,6-10}$ With the right diagnostic imaging and proper treatment, initial improvement may occur within a couple of days. ${ }^{2}$ Symptoms may take from 1 to 3 weeks to completely resolve. ${ }^{1,2,4,6,9,10}$ Resolution of radiographic findings may take weeks to months; however, follow-up imaging is not necessary. ${ }^{4,12}$

\section{Conclusion}

Awareness of the existence of acute calcific tendinitis of the longus colli muscle may be helpful in differentiating this entity from conditions that cause similar severe neck pain. An immunocompromised state may make the diagnosis more difficult, given that abscesses occur more frequently in such conditions. Adalimumab and other medications that may lead to an immunocompromised state may also potentially put patients at high risk for infection and abscess. Our case emphasizes the importance of differentiating the effusion of acute calcific tendinitis from a retropharyngeal abscess. Familiarity with imaging findings may prevent incorrect diagnosis and invasive surgical procedures. Early recognition of the disease facilitates the formation of an early treatment plan, which may lead to rapid recovery and decreased costs.

\section{References}

1. Jimenez S, Millan JM. Calcific retropharyngeal tendinitis: a frequently missed diagnosis. J Neurosurg Spine 2007;6:77-80.

2. Zibis AH, Giannis D, Malizos KN. Acute calcific tendinitis of the longus colli muscle: case report and review of the literature. Eur Spine J 2013;22: $1-5$.

3. Park SY, Jin W, Lee SH, Park JS, Yang DM, Ryn $\mathrm{KN}$. Acute retropharyngeal calcific tendinitis: a case report with unusual location of calcification. Skeletal Radiol 2010;39:817-20.

4. Ellika SK, Payne SC, Patel SC, Jain R. Acute calcific tendinitis of the longus colli: an imaging diagnosis. Dentomaxillofac Radiol 2008;37:121-4.

5. American Society of Health System Pharmacists, Inc. DynaMed. Adalimumab, record no. 232895. Ipswich (MA): EBSCO Information Services; 1995; updated September 7, 2011. Available from: http://search. ebscohost.com/login.asp $x$ ?direct $=$ true\&site $=$ DynaMed \&id=232895. Accessed May 25, 2013.

6. Levy Z, Carroll J, Farley H. Benign nuchal rigidity: the emergency department evaluation of acute prevertebral calcific tendonitis. West J Emerg Med 2012;13:114-6.

7. Vollmann R, Hammer G, Simbrunner J. Pathways in the diagnosis of prevertebral tendinitis. Eur J Radiol 2012;81:114-7.

8. Offiah CE, Hall E. Acute calcific tendinitis of the longus colli muscle: spectrum of CT appearances and anatomical correlation. Br J Radiol 2009;82: e117-21.

9. Shin DE, Ahn CS, Choi JP. The acute calcific prevertebral tendinitis: report of two cases. Asian Spine J 2010;4:123-7.

10. Bladt O, Vanhoenacker P, Bevernage C, Oshoven MV, Hoe LV, D'Haenens P. Acute calcific prevertebral tendinitis. JBR-BTR 2008;91:158-9.

11. Eastwood JD, Hudgins PA, Malone D. Retropharyngeal effusion in acute calcific prevertebral tendinitis: diagnosis with CT and MR imaging. Am J Neuroradiol 1998;19:1789-92.

12. Harnier S, Kuhn J, Harzheim A, Bewermeyer H, Limmroth $\mathrm{V}$. Retropharyngeal tendinitis: a rare differential diagnosis of severe headaches and neck pain. J Head Face Pain 2008;48:158-61. 